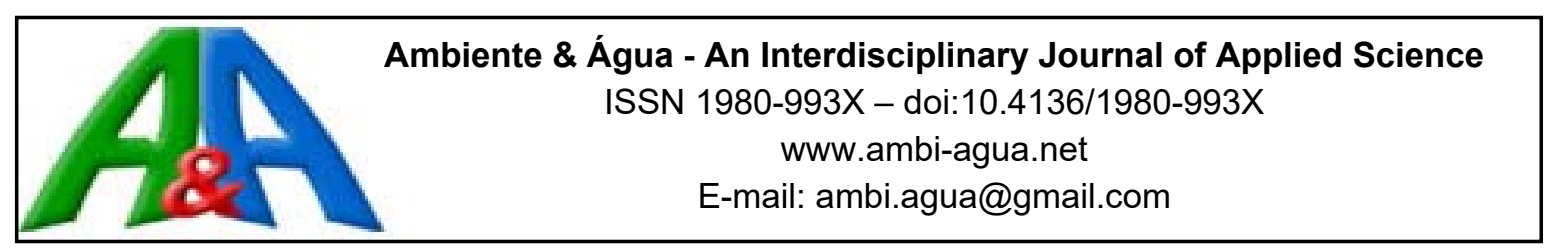

\title{
Variação espacial e sazonal do zooplâncton nos reservatórios do Sistema Cantareira, Brasil
}

\author{
doi:10.4136/ambi-agua.1935
}

Received: 08 May 2016; Accepted: 20 Apr. 2017

\author{
Bruno Paes De-Carli ${ }^{*}$; Júlio César Lopez Doval²; \\ Eduardo Henrique Costa Rodrigues ${ }^{1}$; \\ Marcelo Luiz Martins Pompêo ${ }^{2}$

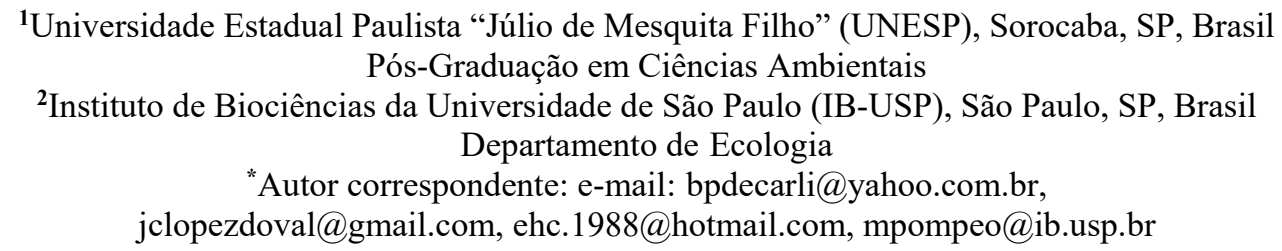

\section{RESUMO}

Neste estudo investigou-se a variação espacial e sazonal da comunidade zooplanctônica e sua relação com as características físicas, químicas e biológicas da água em cinco reservatórios (Jaguari, Jacareí, Cachoeira, Atibainha e Paiva Castro) do sistema Cantareira. Amostragens foram realizadas em maio/junho e novembro/dezembro de $2013 \mathrm{em} 19$ pontos. Para verificar a similaridade dos atributos zooplanctônicos, efetuou-se uma análise de similaridade bifatorial e um ordenamento multidimensional não-métrico. A relação entre os descritores biológicos e ambientais foi examinada usando-se correlação de Pearson. Um total de 35 táxons foi identificado. Os rotíferos predominaram em termos de riqueza e os juvenis de Copepoda seguidos por Bosmina freyi foram os mais abundantes. Em geral, os locais apresentaram condições mesotróficas com exceção do reservatório Jaguari considerado eutrófico. Com base nas análises, foi evidenciado uma variação espaço-sazonal e correspondência entre descritores ambientais e a densidade. A morfometria do reservatório, o tempo de residência, as interações ecológicas e as mudanças de qualidade da água podem ser fatores que explicam a variabilidade observada.

Palavras-chave: bioindicador, comunidade zooplanctônica, limnologia.

\section{Spatial and seasonal variation of zooplankton in reservoirs of the Cantareira system, Brazil}

\begin{abstract}
This study examined the spatial and seasonal variation of zooplanktonic communities and their relationship to the physical, chemical and biological properties of water in five reservoirs (Jaguari, Jacareí, Cachoeira, Atibainha e Paiva Castro) of the Cantareira system. Sampling was performed in May/June and November/December 2013 at 19 sites. To verify similarity of zooplankton attributes, we applied a two-way similarity analysis and a non-metric multidimensional scaling. Relationship between biological and environmental descriptors was examined by Pearson correlation. A total of 35 taxa was identified. Rotifers were predominant
\end{abstract}


in terms of species richness and juvenile copepods followed by Bosmina freyi were the most abundant. Overall, the sites showed mesotrophic conditions, with the exception of the Jaguari reservoir, which was considered eutrophic. Based on the analysis, spatial and seasonal variations and correspondence between environmental indicators and densities were observed. Reservoir morphometry, water retention time, ecological interactions and water quality might explain the observed trend.

Keywords: bioindicator, limnology, zooplankton community.

\section{INTRODUÇÃO}

Reservatórios em particular são ecossistemas artificiais com alto grau de heterogeneidade ambiental (Cardoso-Silva, 2008). Ao longo do eixo central de um reservatório, mudanças físicas, químicas e biológicas ocorrem gradativamente sendo possível observar compartimentos com características lóticas, de transição e lênticas (Thornton et al., 1990). Em todos os ambientes, a análise dos padrões que consideram o espaço e o tempo pode ser utilizada para compreender os processos ecológicos e a complexidade das interações (Fortin e Dale, 2005).

O zooplâncton de ambientes aquáticos continentais é constituído na sua maioria por protozoários, rotíferos, cladóceros e copépodos, os quais desempenham papel importante na cadeia alimentar, transferindo massa e energia de produtores primários para níveis tróficos superiores (Pereira et al., 2011). Nesse grupo, espécies sensíveis à poluição e contaminantes são geralmente eliminadas, e as mais resistentes mostram elevadas taxas de crescimento populacional. Por isso, o zooplâncton pode ser considerado indicador de modificações causadas nos ecossistemas aquáticos por atividades impactantes (Barbosa et al., 2006; Galdino et al., 2007; Santos et al., 2009).

Pesquisas sobre a comunidade zooplanctônica são realizadas desde a década de 70 no Brasil (Nordi e Watanabe, 1978; Corgosinho e Pinto-Coelho, 2006; Santos-Wisniewski e Rocha, 2007; Almeida et al., 2009; Sartori et al., 2009; Paranhos et al., 2013; Dantas-Silva e Dantas, 2013). Dentre os trabalhos realizados no estado de São Paulo destacam-se: levantamento de espécies (Sendacz e Kubo, 1982; Matsumura-Tundisi e Silva, 2002; Matsumura-Tundisi e Tundisi, 2011; Silva e Matsumura-Tundisi, 2011; Souza-Soares et al., 2011), qualidade ambiental (Sendacz e Santos-Soares, 2006) e migração vertical e horizontal (Casanova e Henri, 2004).

O sistema Cantareira é um complexo de represas destinado ao abastecimento público de mais da metade da região Metropolitana de São Paulo (Whately e Cunha, 2007). Devido ao quadro atual de crise hídrica, são necessários levantamentos com intuito de descrever a situação desses reservatórios e identificar possíveis mudanças nas condições ambientais. Desse modo, objetivou-se com este trabalho determinar a variação espacial e sazonal do zooplâncton e sua relação com os parâmetros limnológicos em cinco reservatórios do sistema Cantareira.

\section{MATERIAIS E MÉTODOS}

\section{1. Área de estudo}

Localizado na região norte da Grande São Paulo, o sistema Cantareira produz 33 mil litros de água por segundo e é composto por cinco reservatórios (Jaguari, Jacareí, Cachoeira, Atibainha e Paiva Castro) de regularização de vazões com túneis e canais de interligação para transferência de água de uma represa para outra (Tabela 1, Figura 1). Esse complexo possui ainda um reservatório destinado a manter o fluxo para uma estação de tratamento, o reservatório Águas Claras. O sistema compreende as bacias hidrográficas do alto rio Tietê e do Piracicaba. O clima da região é subtropical de altitude com inverno seco e verão ameno. No entorno, a 
vegetação original pertence ao bioma de mata atlântica, e atualmente, consiste em mata secundária (Oliveira et al., 2008).

Tabela 1. Características hidrológicas das represas do Sistema Cantareira.

\begin{tabular}{lcccc}
\hline Informações/ Mananciais & Jaguari/Jacareí & Cachoeira & Atibainha & Paiva Castro \\
\hline Área de drenagem $\left(\mathrm{km}^{2}\right)$ & $1027 / 203$ & 392 & 312 & 369 \\
Início de operação & 1982 & 1974 & 1975 & 1973 \\
Volume útil $\left(\mathrm{hm}^{3}\right)$ & 808,12 & 69,75 & 95,26 & 7,61 \\
Vazão média $\left(\mathrm{m}^{3} / \mathrm{s}\right)$ & 25,2 & 8,5 & 6,0 & 4,6 \\
Cota altimétrica (m) & 844,0 & 821,88 & 786,72 & 745,61 \\
Tempo de retenção (dias) & 368,5 & 40,1 & 105,8 & 10,7 \\
Sub-bacias hidrográficas & Rio Jaguari/Jacareí & Rio Cachoeira & Rio Atibainha & Rio Juquery \\
\hline
\end{tabular}

Fonte: Whatley e Cunha (2007); Netto e Barros (2010).

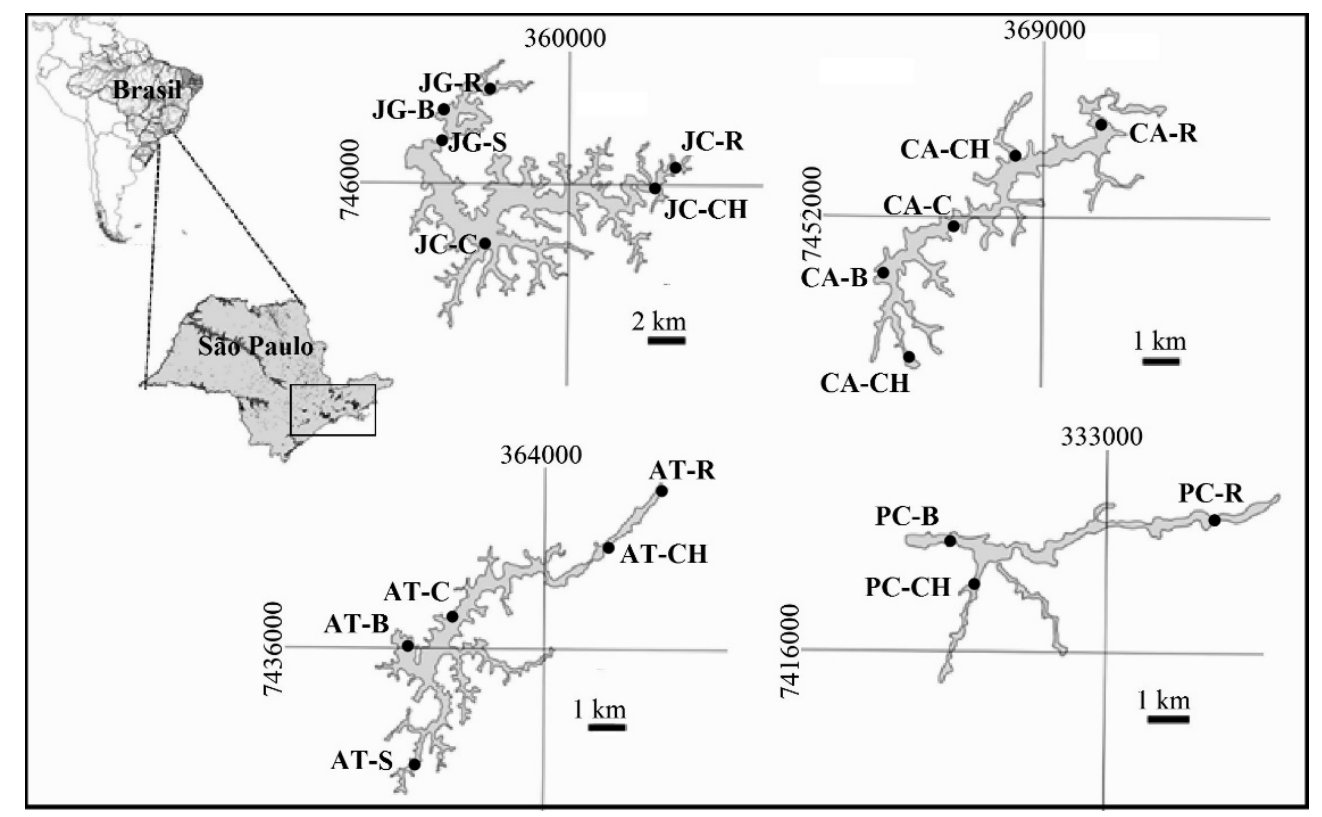

Figura 1. Pontos de amostragem nos reservatórios do sistema Cantareira. Legenda: Jaguari (JG), Jacareí (JC), Cachoeira (CA), Atibainha (AT) e Paiva Castro (PC). Região central $(\mathrm{C})$, canais $(\mathrm{CH})$, saída d'água $(\mathrm{S})$, barragem $(\mathrm{B})$ e rio $(\mathrm{R})$.

\subsection{Procedimento de coleta e análise}

As coletas foram realizadas nas represas Jaguari, Jacareí, Cachoeira, Atibainha e Paiva Castro no inverno (maio/junho) e verão (novembro/dezembro) de 2013. Em cada local, amostras integradas da coluna d'água foram coletadas por meio de mangueiras de diferentes comprimentos $(5,3$ e $2 \mathrm{~m})$ conforme a profundidade máxima mensurada.

Em laboratório, o material foi filtrado para as determinações de clorofila a, sólidos em suspensão, nitrato, nitrito e íon amônio. Em amostras de água bruta foram determinadas as concentrações de fósforo total (Tabela 2). Em campo foram tomadas medidas da profundidade máxima (m), do disco de Secchi $(\mathrm{m})$ além do oxigênio dissolvido $\left(\mathrm{mg} \mathrm{L}^{-1}\right)$, temperatura $\left({ }^{\circ} \mathrm{C}\right)$, condutividade elétrica $(\mu \mathrm{S} . \mathrm{cm})$ e $\mathrm{pH}$ medidos por meio de sonda multiparâmetros (YSI mod 556 MPS). 
Amostras do zooplâncton foram coletadas com rede de $68 \mu \mathrm{m}$ através de arrasto vertical seguindo a coleta d'água. Os organismos foram anestesiados com água gaseificada e em seguida fixados com solução de formol 4\% com sacarose (Cetesb, 1990). A contagem dos organismos foi realizada por meio de uma câmara de Sedgwick-Rafter. A densidade numérica foi expressa em organismos por metro cúbico (org. $/ \mathrm{m}^{3}$ ) (Cetesb, 1990). A identificação taxonômica foi baseada em Koste (1978), Reid et al. (1988), Shiel e Koste (1992), Nogrady et al. (1995), Segers (1995), Segers e Dumont (1995), De Smet (1996), Elmoor-Loureiro (1997), Rocha et al. (2011), Nogrady e Segers (2002), Kotov et al. (2003), Silva (2003), Elmoor-Loureiro et al. (2004), Gützel et al. (2004), Kotov (2009) e Perbiche-Neves et al. (2015).

Tabela 2. Parâmetros limnológicos e metodologias empregadas.

\begin{tabular}{lll}
\hline \multicolumn{1}{c}{ Variáveis } & \multicolumn{1}{c}{ Métodos } & \multicolumn{1}{c}{ Referências } \\
\hline Sólidos em suspensão $\left(\mathrm{mg} \mathrm{L}^{-1}\right)$ & Gravimétrico & Teixeira et al. (1965) \\
Fósforo total $\left(\mu \mathrm{g} \mathrm{L}^{-1}\right)$ & Espectrofotométrico & Valderrama (1981) \\
Nitrato $\left(\mu \mathrm{g} \mathrm{L}^{-1}\right)$ & Espectrofotométrico & Mackereth et al. (1978) \\
Nitrito $\left(\mu \mathrm{g} \mathrm{L}^{-1}\right)$ & Espectrofotométrico & Mackereth et al. (1978) \\
Amônio $\left(\mu \mathrm{g} \mathrm{L}^{-1}\right)$ & Espectrofotométrico & Koroleff (1976) \\
Nitrogênio inorgânico dissolvido (DIN) $\left(\mu \mathrm{g} \mathrm{L}^{-1}\right)$ & $\sum \mathrm{NO}_{2}+\mathrm{NO}_{3}+\mathrm{NH}_{4}$ & - \\
Clorofila a $\left(\mu \mathrm{g} \mathrm{L}^{-1}\right)$ & Espectrofotométrico & Lorenzen $(1967)$ \\
\hline
\end{tabular}

Fonte: Teixeira et al. (1965); Valderrama (1981); Mackereth et al. (1978); Koroleff (1976); Lorenzen (1967).

\subsection{Análise de dados}

O índice do estado trófico (IET) foi calculado com base na média aritmética dos dados de fósforo total e clorofila a, conforme Carlson (1977) e modificado por Lamparelli (2004) para reservatórios. A seguinte classificação foi utilizada: IET $<=47$ (ultraoligotrófico), $47<$ IET $<=52$ (oligotrófico), $52<$ IET $<=59$ (mesotrófico), $59<$ IET $<=63$ (eutrófico), $63<$ IET $<=67$ (supereutrófico) e IET $>67$ (hipereutrófico).

Para padronização das variáveis limnológicas às análises estatísticas, utilizou-se o método que calcula a razão entre as diferenças dos valores brutos menos os mínimos divididos, pela diferença entre o máximo e mínimo (Milligan e Cooper, 1988). Para o zooplâncton, os dados foram logaritimizados $(\log X+1)$ (Melo e Hepp, 2008).

Com intuito de visualizar o ordenamento espacial, aplicou-se uma análise de escalonamento não-métrico multidimensional (nMDS) para as matrizes de densidade e riqueza por estação sazonal (Melo e Hepp, 2008). Para detectar a significância do ordenamento, efetuou-se uma análise de similaridade bifatorial (ANOSIM) (Clark, 1993). Para a matriz de riqueza, utilizou-se o índice de similaridade de Jaccard, e para abundância aplicou-se o índice de Bray-Curtis. A correlação de Pearson (r) foi realizada para analisar as relações entre os descritores ambientais e a densidade. As análises foram realizadas com nível de significância de $0,05(95 \%)$.

\section{RESULTADOS E DISCUSSÃO}

\subsection{Variáveis limnológicas}

Os parâmetros limnológicos mensurados nos reservatórios estão descritos na Tabela 3. Maiores profundidades foram verificadas no reservatório Jaguari $(29,1 \mathrm{~m})$ e locais mais rasos no reservatório Paiva Castro $(9,2 \mathrm{~m})$. Maior transparência foi observada no reservatório 
Cachoeira $(2,8 \mathrm{~m})$ em contraste com o Jaguari $(0,5 \mathrm{~m})$. Temperaturas mais altas foram registradas na represa Jacareí $\left(25,6^{\circ} \mathrm{C}\right)$ no verão, com águas mais frias no reservatório Atibainha $\left(18,8^{\circ} \mathrm{C}\right)$ no inverno. Maiores teores de oxigênio dissolvido foram mensurados no reservatório Cachoeira $\left(9,9 \mathrm{mg} \mathrm{L}^{-1}\right)$ no inverno e baixas concentrações na represa Jaguari $\left(6,0 \mathrm{mg} \mathrm{L}^{-1}\right)$ no verão. A condutividade elétrica não variou significativamente entre as localidades, com valores entre 30,9 $\mu \mathrm{S} / \mathrm{cm}$ (Atibainha) e 36,0 $\mu \mathrm{S} / \mathrm{cm}$ (Jacareí).

Tabela 3. Médias das variáveis limnológicas dos reservatórios do Sistema Cantareira obtidas no verão (V) e inverno (I) durante 2013. Legendas: ultraoligotrófico (U), oligotrófico (O), mesotrófico (M), eutrófico (E), supereutrófico $(\mathrm{S})$ e hipereutrófico $(\mathrm{H})$.

\begin{tabular}{|c|c|c|c|c|c|c|c|c|c|c|}
\hline \multirow{2}{*}{ Variáveis } & \multicolumn{2}{|c|}{ Jaguari } & \multicolumn{2}{|c|}{ Jacareí } & \multicolumn{2}{|c|}{ Cachoeira } & \multicolumn{2}{|c|}{ Atibainha } & \multicolumn{2}{|c|}{ Paiva Castro } \\
\hline & I & V & I & V & I & V & I & V & I & V \\
\hline Profundidade (m) & 29,1 & 21,9 & 18,5 & 16,2 & 13,6 & 12,6 & 13,7 & 12,3 & 9,8 & 9,2 \\
\hline Nitrito $\left(\mu \mathrm{g} \mathrm{L}^{-1}\right)$ & 2,0 & 26,8 & 1,6 & 8,3 & 0,7 & 3,7 & 1,2 & 4,5 & 1,4 & 4,3 \\
\hline Nitrato $\left(\mu \mathrm{g} \mathrm{L}^{-1}\right)$ & 358,7 & 686,2 & 323,7 & 417,8 & 346,0 & 330,4 & 257,2 & 228,8 & 233,4 & 219,3 \\
\hline Amônio $\left(\mu \mathrm{g} \mathrm{L}{ }^{-1}\right)$ & 10,1 & 119,1 & 18,6 & 41,4 & 14,2 & 21,7 & 26,7 & 17,8 & 29,8 & 17,7 \\
\hline $\operatorname{DIN}\left(\mu \mathrm{g} \mathrm{L}{ }^{-1}\right)$ & 370,8 & 832,1 & 344,0 & 466,6 & 361,0 & 355,9 & 285,1 & 251,2 & 264,6 & 724,3 \\
\hline Fósforo total $\left(\mu \mathrm{g} \mathrm{L}{ }^{-1}\right)$ & 32,4 & 16,4 & 8,5 & 6,1 & 6,3 & 4,5 & 4,5 & 4,5 & 7,2 & 4,5 \\
\hline Clorofila a $\left(\mu \mathrm{g} \mathrm{L}^{-1}\right)$ & 9,5 & 11,2 & 2,5 & 6,0 & 1,1 & 1,7 & 1,8 & 1,9 & 1,6 & 1,9 \\
\hline Disco de secchi (m) & 2,3 & 0,5 & 3,0 & 1,6 & 2,8 & 2,4 & 2,3 & 2,4 & 1,9 & 2,5 \\
\hline $\begin{array}{l}\text { Sólidos em suspensão } \\
\left(\mathrm{mg} \mathrm{L}^{-1}\right)\end{array}$ & 5,6 & 16,2 & 2,0 & 7,0 & 2,2 & 3,4 & 1,7 & 1,7 & 3,3 & 2,8 \\
\hline Temperatura $\left({ }^{\circ} \mathrm{C}\right)$ & 18,9 & 24,3 & 19,6 & 25,6 & 19,9 & 23,5 & 18,8 & 25,0 & 19,4 & 23,1 \\
\hline $\begin{array}{l}\text { Condutividade elétrica } \\
(\mu \mathrm{S} / \mathrm{cm})\end{array}$ & 33,8 & 33,6 & 34,3 & 36,0 & 32,1 & 32,9 & 30,9 & 31,0 & 34,0 & 35,0 \\
\hline $\mathrm{pH}$ & 8,30 & 6,7 & 7,0 & 6,8 & 6,6 & 6,1 & 6,40 & 6,5 & 6,6 & 7,6 \\
\hline $\begin{array}{l}\text { Oxigênio dissolvido } \\
\left(\mathrm{mg} \mathrm{L}^{-1}\right)\end{array}$ & 9,5 & 6,0 & 7,1 & 7,2 & 9,9 & 9,3 & 8,14 & 7,9 & 7,3 & 8,0 \\
\hline IET & 62 & 60 & 54 & 56 & 52 & 52 & 52 & 53 & 53 & 53 \\
\hline Estado trófico & $\mathrm{E}$ & $\mathrm{E}$ & M & M & M & M & M & M & M & M \\
\hline
\end{tabular}

Em relação aos nutrientes (fósforo total, nitrato, nitrito, amônio e nitrogênio inorgânico dissolvido), clorofila a e sólidos em suspensão, foram determinadas concentrações elevadas no reservatório Jaguari. Nesse reservatório foram observadas condições eutróficas, em contraste com os demais, considerados mesotróficos. Em estudo realizado por Gazonato-Neto et al. (2014) em dois mananciais do sistema Cantareira, pode-se notar que o reservatório Jaguari encontrava-se mais eutrofizado do que o Jacareí.

\subsection{Comunidade zooplanctônica}

Um total de 35 táxons foi identificado, sendo 17 rotíferos, 10 copépodes e 8 cladóceros (Tabela 4).

Possivelmente, os rotíferos predominaram por serem animais oportunistas e geralmente associados a ambientes eutrofizados (Rocha et al., 1999). Além disso, registrou-se a presença do rotífero exótico K. bostoniensis que foi recentemente introduzido no Brasil (Landa et al., 2002). Esse braquionídeo pode representar risco por competir com espécies nativas, ocasionando modificações na estrutura e dinâmica das comunidades (Bomfim et al., 2016). 
Tabela 4. Lista de táxons encontrados nos reservatórios do sistema Cantareira em 2013.

\begin{tabular}{ll}
\hline \multicolumn{1}{c}{ Táxons } & \multicolumn{1}{c}{ Rotifera } \\
\hline Calanoida & Ascomorpha sp. Harring, 1913 \\
\hline Copepodito & Asplanchna sp. Eckstein, 1883 \\
Nauplios & Asplanchna brightwellii Gosse, 1850 \\
Notodiaptomus cearensis (Wright, 1936) & Brachionus falcatus Zacharias 1898 \\
Notodiaptomus jatobensis (Wright, 1936) & Collotheca Harring, 1913 \\
Cyclopoida & Conochilus dossuarius Hudson, 1885 \\
Copepodito & Conochilus unicornis Rousselet, 1892 \\
Mesocyclops longisetus (Thiebaud, 1912) & Filinia opoliensis (Zacharias, 1898) \\
Nauplios & Kellicottia bostoniensis (Rousselet,1908) \\
Thermocyclops decipiens (Kiefer, 1929) & Keratella americana Carlin,1943 \\
Thermocyclops minutus (Lowndes, 1934) & Keratella cochlearis (Gosse,1851) \\
Harpacticoida não identificado & Lecane sp. Nitzsch, 1827 \\
Cladócera & Polyarthra vulgaris Carlin,1943 \\
Bosmina freyi De Melo \& Hebert, 1994 & Ptygura melicerta Ehrenberg 1832 \\
Bosminopsis deitersi Richard, 1895 & Synchaeta stylata Wierzejski, 1893 \\
Ceriodaphnia cornuta rigaudi (Sars,1886) \\
Ceriodaphnia dubia Richard, 1894 & Synchaeta sp. Ehrenberg, 1832 \\
Ceriodaphnia richardi Sars, 1901 & Trichocerca capucina (Wierzejski \& Zacharias, 1893) \\
Ceriodaphnia silvestrii Daday, 1902 & \\
Daphnia gessneri (Herbst, 1967) & \\
Diaphanosoma spinulosum Herbst, 1975 & \\
\hline
\end{tabular}

No inverno, maiores riquezas foram assinaladas na barragem do reservatório Jaguari e no centro da represa Cachoeira com 17 espécies (Figura 2A). Nessa estação, foi observado menor riqueza na barragem do reservatório Atibainha (4 táxons). Na amostragem de verão, o maior número de espécies foi encontrado na saída de água da represa Jaguari (25 táxons) em contraste com a entrada do rio no reservatório Paiva Castro (4 táxons) (Figura 2B).

A distância entre os corpos d'água e os tipos de conexão que apresentam com o rio e com todo o sistema podem ser fatores determinantes para a composição de espécies. Características locais e próprias de cada área envolvem interações bióticas e abióticas que interferem na dinâmica de cada comunidade moldando sua estrutura (Abra et al., 2014).

Durante o inverno, a densidade total variou de 218 a 12.200 ind. $/ \mathrm{m}^{3}$, enquanto no verão, os valores oscilaram entre 131 e 10.143 ind. $\mathrm{m}^{3}$ (Figura 3A e B). Nos dois períodos estudados, os copepoditos de Calanoida e Cyclopoida seguidos por Bosmina freyi foram mais abundantes. A produção de estágios jovens (copepoditos) pode ser considerada uma estratégia adaptativa das populações para compensar a alta mortalidade dos indivíduos antes de alcançarem a fase adulta (Espíndola et al., 2000). Em relação a B. freyi, é reconhecido que este cladócero pode 
indicar ambientes eutrofizados (Rocha et al., 1999; Güntzel et al., 2004; Silva et al., 2011; Gazonato-Neto, 2013).

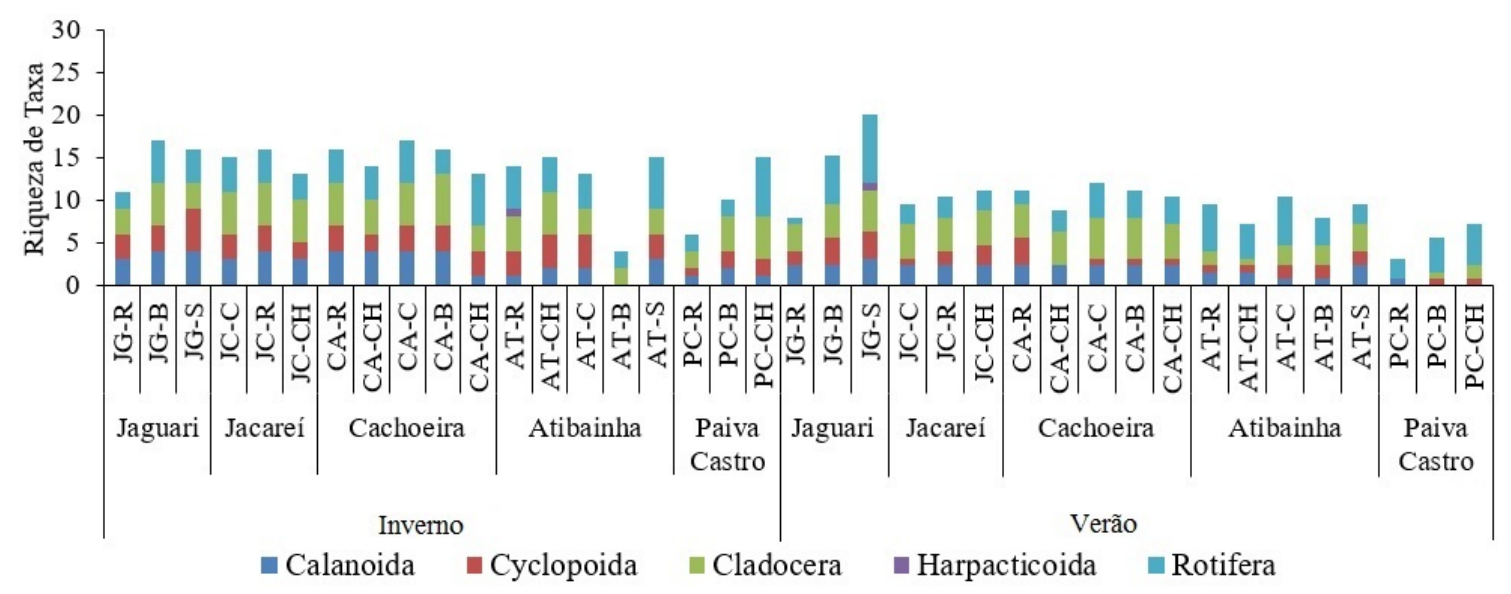

Figura 2. Riqueza dos grupos zooplanctônicos em cinco reservatórios do sistema Cantareira no inverno e verão de 2013.

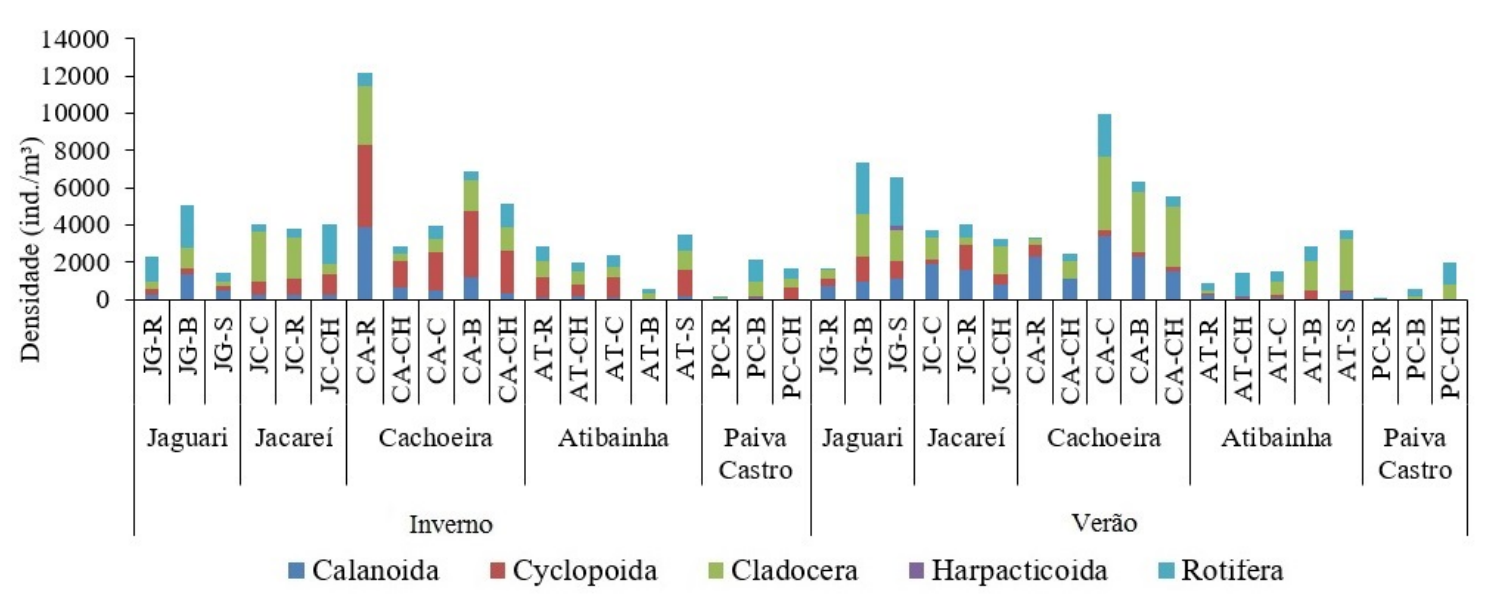

Figura 3. Densidade dos grupos zooplanctônicos (ind. $/ \mathrm{m}^{3}$ ) em cinco reservatórios do sistema Cantareira no inverno e verão de 2013.

Através da nMDS foi possível observar agrupamentos e heterogeneidade espacial (Figura 4), confirmados pela ANOSIM para os dados de riqueza (espacial $=0,37 \mathrm{p}<0,05$; sazonal $=0,31 ; p<0,05)$ e densidade $($ espacial $=0,74 p<0,05$; sazonal $=0,50 ; p<0,05)$. Nesse sentido, variações físico-químicas e biológicas no ambiente podem resultar em alterações na dinâmica do zooplâncton (Abra et al., 2014). A hidrodinâmica também influencia os fatores limnológicos e consequentemente as populações aquáticas. Brook e Woodward (1956) observaram que o tempo de residência da água acima de 18 dias propicia o recrutamento de planctontes. Enquanto Melão (2010) analisou que o tempo de residência deve ser superior a 16 dias para o desenvolvimento de microcrustáceos. O baixo tempo de residência calculado para represa Paiva Castro (10 dias) pode ter sido um dos principais fatores que influenciaram a baixa riqueza no local.

Cabe ressaltar que a heterogeneidade ambiental não está relacionada somente a fatores como o regime operacional influenciado pelas demandas de água, mas também das características fisiográficas e atividades humanas que se desenvolvem ao longo de bacias hidrográficas (Hackbart et al., 2015). Fatores como qualidade e quantidade de recursos 
alimentares, competição intra e interespecífica, parasitismo e predação também devem ser considerados (Xie et al., 1998; Ortega-Mayagoitia et al., 2011; Meirinho et al., 2013). Assim, existem múltiplos elementos envolvidos na heterogeneidade dos ecossistemas aquáticos e todos esses podem agir conjuntamente (Shimabukuro, 2014).
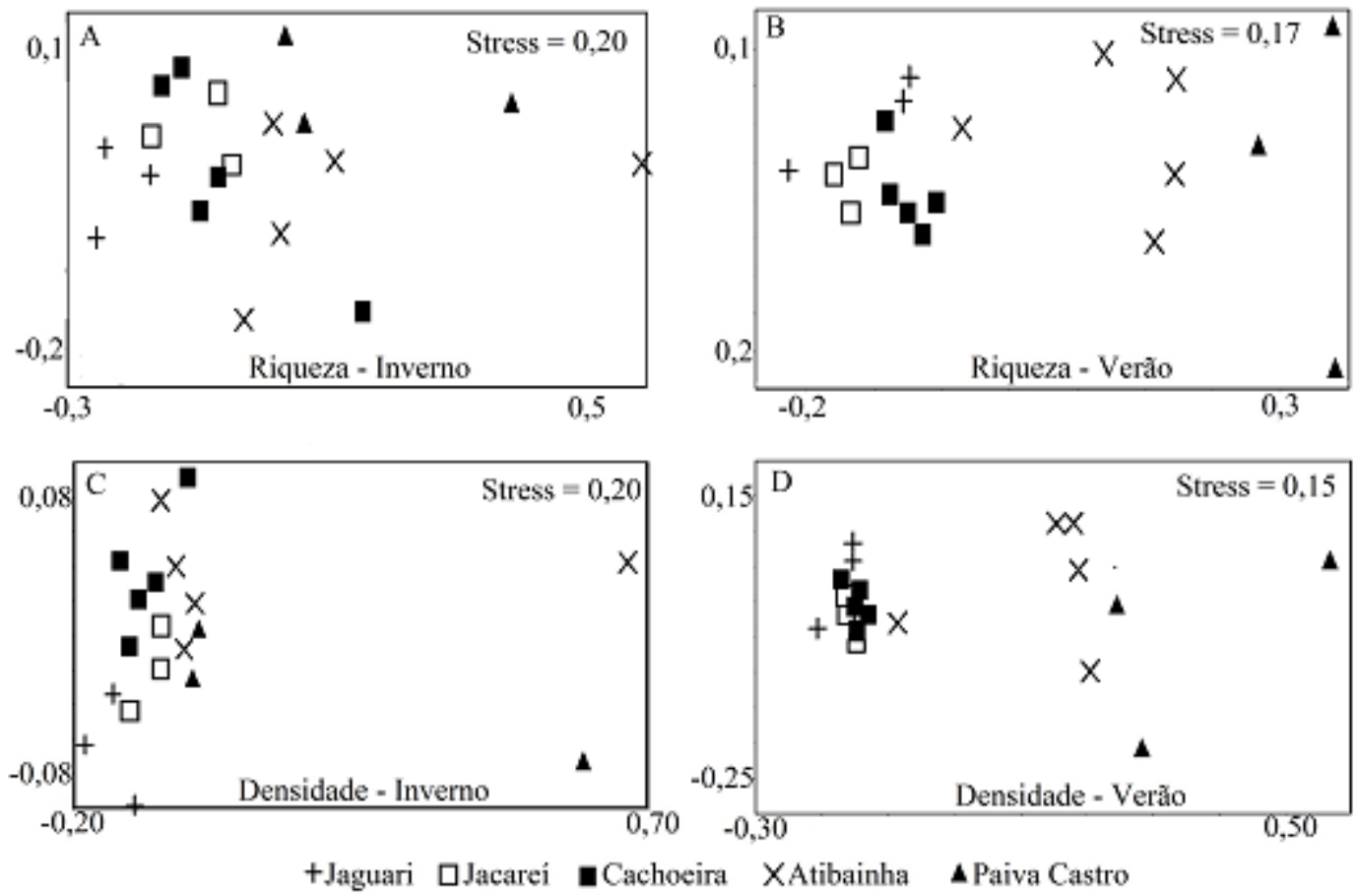

Figura 4. Diagramas de ordenação não-métrica multidimensional (nMDS) da composição e abundância de espécies do sistema Cantareira em 2013.

Os dados de densidade de Calanoida estiveram correlacionados positivamente com a concentração de nitrogênio inorgânico dissolvido $(r=0,39 \mathrm{p}<0,05)$. De acordo com Panosso et al. (2003), essa associação pode estar relacionada ao hábito alimentar do crustáceo Notodiaptomus, que pode ingerir cianobactérias frequentemente encontradas em locais com enriquecimento de nitrogênio. A associação dos rotíferos $(\mathrm{r}=0,36 \mathrm{p}<0,05)$ e cladóceros $(\mathrm{r}=0,45 \mathrm{p}<0,05)$ com a profundidade, pode estar relacionada com a quantidade de sólidos em suspensão e uma possível preferência dos organismos pela zona litoral do corpo d'água. Em geral, na região litoral de lagos e reservatórios ocorrem macrófitas aquáticas que podem servir como abrigo para os animais (Sorf e Devetter, 2011). Com relação aos sólidos, a concentração da fração inorgânica pode ser prejudicial, uma vez que partículas podem aderir-se e obstruir os aparatos dos organismos. Por outro lado, altas concentrações da fração orgânica podem ser mais nutritivas (Mortari, 2004; Hoffmann, 2012). A densidade dos Rotifera ( $\mathrm{r}=0,32 \mathrm{p}<0,05$ ) apresentou correlação positiva com o fósforo total, corroborando outras pesquisas que apontam esse grupo como indicador de águas eutrofizadas (Landa et al., 2002; Eler et al., 2003).

\section{CONCLUSÃO}

Em relação a qualidade da água, pode-se observar locais mesotróficos, com exceção do reservatório Jaguari com elevada trofia. Diante disso, faz-se necessário adotar medidas de saneamento ambiental com intuito de reduzir a carga de nutrientes no sistema. Quanto a comunidade zooplanctônica, os Rotifera predominam em número de espécies e os Copepoda em abundância. As populações apresentam heterogeneidade espacial e sazonal, sendo essa variação relacionada a fatores aqui discutidos. Futuros estudos sobre os ovos de resistência (diapausa) serão úteis para compreender a dispersão dos organismos entre os reservatórios 
interligados. As variáveis bióticas e abióticas estiveram relacionadas, sustentando o potencial do zooplâncton como ferramenta indicadora de qualidade ambiental.

\section{AGRADECIMENTOS}

Ao auxílio financeiro concedido pela Fundação de Amparo à Pesquisa do Estado de São Paulo FAPESP (Convênio CAPES), processos 2012/11890-4 e 2014/04471-0.

\section{REFERÊNCIAS}

ABRA, J.; CASTILHO-NOLL, M.S.M., STEPHAN, L.R.; HENRY, R. Estudo de longo prazo do zooplâncton em lagoas marginais ao rio Paranapanema. In: HENRY, R. Represa de Jurumirim: ecologia, modelagem e aspectos sociais. Ribeirão Preto: Holos, 2014. 435p.

ALMEIDA, V. L. S.; DANTAS, Ê. W.; MELO-JÚNIOR, M.; BITTENCOURT-OLIVEIRA, M. C.; MOURA, A. N. Zooplanktonic community of six reservoirs in northeast Brazil.

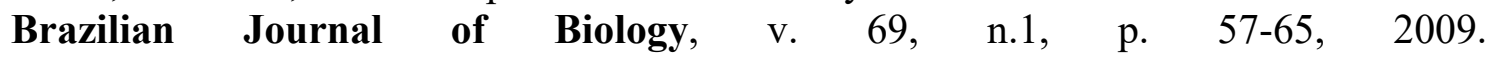
http://dx.doi.org/10.1590/S1519-69842009000100007

BARBOSA, P.M.M.; BRITO, S.; RIETZLER, A.C. Diversidade do zooplâncton de Minas Gerais. Revista Ciência Hoje, v. 38, n. 227, 2006.

BOMFIM, F. F.; MANTOVANO, T.; SCHWIND, L. T. F.; PALAZZO, F.; BONECKER, C. C.; LANSAC-TÔHA, F. A. Geographical spread of the invasive species Kellicottia longispina (Kellicott, 1879) and K. bostoniensis (Rousselet, 1908): A scientometric approach. Acta Scientiarium Biological Sciences, v.38, n.1, p. 29-36, 2016. http://dx.doi.org/10.4025/actascibiolsci.v38i1.28252

BROOK, A.J.; WOODWARD, W.B. Some observations on the effects of water inflow and outflow on the plankton of small lakes. The journal of Animal Ecology, v. 25, n. 1, p 2235, 1956. http://www.jstor.org/stable/1848

CARLSON, R.E. A trophic state index for lakes. Limmology and Oceanography, v. 22, n. 2, p. 361-380, 1977. http://dx.doi.org/10.4319/1o.1977.22.2.0361

CASANOVA, S. M.; HENRY, R. Longitudinal distribuition of Copepoda population in the transition zone of Paranapanema River and Jurumim Reservoir (São Paulo, Brasil) (São Paulo, Brazil) and interchange with two lateral lakes. Brazilian Journal of Biology, v.54, n.1, p.11-26, 2004. http://dx.doi.org/10.1590/S1519-69842004000100003

CARDOSO-SILVA, S. Heterogeneidade espacial e a qualidade das águas superficiais do reservatório Guarapiranga (São Paulo - SP - Brasil). 2008. 99p. Dissertação (Mestrado em Ecologia) - Instituto de Biociências, Universidade de São Paulo, São Paulo, 2008.

CLARKE, K. R. Non-parametric multivariate analysis of changes in community structure. Australian Journal of Ecology, v.18, p. 117-143, 1993. http://dx.doi.org/10.1111/j.1442-9993.1993.tb00438.x

COMPANHIA DE TECNOLOGIA DE SANEAMENTO AMBIENTAL - CETESB. Norma Técnica L5.314 - Métodos de coleta de zooplâncton marinho e de água doce. São Paulo, 1990. 24p. 
CORGOSINHO, P.H.C.; PINTO-COELHO, R.M. Zooplankton biomass, abundance and allometric patterns along an eutrophic gradient at Furnas Reservoir (Minas Gerais, Brazil). Acta Limnologica Brasiliensia, v.18, n.2, p. 213-224, 2006.

DANTAS-SILVA, L.T.; DANTAS, E.W. Zooplâncton e a eutrofização em reservatórios do nordeste brasileiro. Oecologia Australis, v.17, n.2, p. 53- 58, 2013. http://dx.doi.org/10.4257/oeco.2013.1702.06

DE SMET, W. H. Rotifera: The Proalidae (Monogononta). In: DUMONT, H. J. (ed). Guides to the Identification of the microinvertebrates of the continental waters of the world. The Hague: SBP Academic Publishing, 1996. v.4, p. 102.

ELER, M.N.; PARESCHI, D.C.; ESPÍNDOLA, E.L.G.; BARBOSA, D.S. Ocorrência de Rotifera e sua relação com o estado trófico da água em pesque-pague na bacia do rio Mogi-Guaçu - SP. Boletim Técnico do CEPTA-Pirassununga, v.16, p.41-56, 2003.

ELMOOR-LOUREIRO, L. M. A. Manual de identificação de cladóceros límnicos do Brasil. Brasília: Universa, 1997. p.156.

ELMOOR-LOUREIRO, L. M. A.; MENDONÇA-GALVÃO, L.; PADOVESIFONSECA, C. New cladoceran records from lake Paranoá, Central Brazil. Brazilian Journal of Biology, v. 43, n. 3a, p. 415-422, 2004. http://dx.doi.org/10.1590/S151969842004000300006

ESPÍNDOLA, E. L. G.; MATSUMURA-TUNDISI, T.; RIETZLER, A.C.; TUNDISI, J. G. Spatial heterogeneity of the Tucuruí reservoir (State of Pará, Amazonia, Brazil) and the distribution of zooplâncton species. Revista Brasileira de Biologia, v.60, n.2, p. 179193, 2000. http://dx.doi.org/10.1590/S0034-71082000000200001

FORTIN, M.J.; DALE, M.R.T. Spatial Analys: a guide for ecologists. Cambridge: University Press, 2005. 358p.

GALDINO, T.S.; GUSMÃO, L.M.O.; NEUMANN-LEITÃO, S.; SILVA, T.A.; SCHWAMBORN, R.; OLIVEIRA, G.C. Zooplâncton como indicador da qualidade ambiental nas desembocaduras norte e sul do canal de Santa Cruz - Itamaracá - PE Brasil. In: CONGRESSO DE ECOLOGIA DO BRASIL, 23 a 28 de Setembro de 2007, Caxambu - MG. Anais... São Paulo: SEB, 2007.

GAZONATO-NETO, A. J. Estudo limnológico dos reservatórios Jaguari e Jacareí com ênfase na comunidade zooplanctônica. 2013. 115 f. Dissertação (Mestrado em Ecologia e Recursos Naturais) - Universidade Federal de São Carlos, São Carlos, 2013.

GAZONATO-NETO, A.J.; SILVA, L.C.; SAGGIO, A.A.; ROCHA, O. Zooplankton communities as eutrophication bioindicators in tropical reservoirs. Biota Neotropica, v.14, n.4, p.1-12, 2014. http://dx.doi.org/10.1590/1676-06032014001814

GÜTZEL, A. M.; MATSUMURA-TUNDISI, T.; ROCHA, O. Macrothrix flabelligera, a newly-recorded Cladocera Macrothricidae in Brazilian freshwaters. Brazilian Journal of Biology, v.64, n.2, p.221-226, 2004. http://dx.doi.org/10.1590/S151969842004000200006 
HACKBART, V.C.S.; MARQUES, A.R.P.; KIDA, B.M.S.; TOLUSSI, C.E.; NEGRI, D.D.B.; MARTINS, I.A. et al. Avaliação expedita da heterogeneidade espacial horizontal intra e inter reservatórios do sistema Cantareira (Represas Jaguari-Jacareí, São Paulo). In: POMPÊO, M. et al. (Orgs.) Ecologia de reservatórios e interfaces. São Paulo: Instituto de Biociências da Universidade de São Paulo, 2015.

HOFFMAN, P. Análise temporal da composição, abundância, diversidade dos Cladocera e crescimento alométrico de Daphnia spp. O. F. Müeller, 1785 na Lagoa do Camargo, lateral ao Rio Paranapanema em sua zona de desembocadura na Represa de Jurumirim. 2012. 158f. (Doutorado em Zoologia) - Instituto de Biociências da Universidade Estadual Paulista Júlio de Mesquita Filho, Botucatu, 2012.

KOROLEFF, F. Determination of nutrients. In: GRASSHOFF, K. (Ed.). Methods of seawater analysis. Weinheim: Verlag Chemie Weinhein, 1976. p.117-181.

KOSTE, W. Rotatoria, die Rädertiere Mitteleuropas: Überordnung Monogononta: ein Bestimmungswerk. Berlin: Gebrüder Borntraeger, 1978.

KOTOV, A. A. A revision of Leydigia Kurz, 1875 (Anomopoda, Cladocera, Branchiopoda), and subgeneric differentiation within the genus. Zootaxa, v. 2082, 2009. http://dx.doi.org/10.11646/zootaxa.3814.3.7

KOTOV, A. A.; DAMME, K. V.; ELÍAS-GUTIÉRREZ. Differentiation between African Leydigia ciliate Gauthier, 1939 and Neotropical L. cf. striata Birabén, 1939 (Chydoridae, Anomopoda, Cladocera). Hydrobiologia, v. 505, p.179-197, 2003.

Lamparelli, M. C. Grau de Trofia em Corpos D’Água do Estado de São Paulo: Avaliação dos Métodos de Monitoramento. 2004. 238f. Tese (Doutorado) - Instituto de Biociências, Universidade de São Paulo, São Paulo, 2004.

LANDA, G.G.; AGUILA, L.M.R Del; PINTO-COELHO, R. Distribuição espacial e temporal de Kellicottia bostoniensis (Rousselet, 1908) (Rotifera) em um grande reservatório tropical (reservatório de Furnas), Estado de Minas Gerais, Brasil. Acta Scientiarum, v. 24, n. 2, p. 313-319, 2002. http://dx.doi.org/10.4025/actascibiolsci.v24i0.2272

LORENZEN, C. J. Determination of chlorophyll and pheo-pigments:spectrophotometric equations. Limnology Oceanography, v. 12, n. 2, p.343-346, 1967. http://dx.doi.org/10.4319/1o.1967.12.2.0343

MACKERETH, F. J. H.; HERON, J.; TALLING, J. F. Water analysis: some revised methods for limnologists. Dorset: Freshwater Biol. Ass., 1978. 121p.

MATSUMURA-TUNDISI, T.; SILVA, W. M. Occurrence of Mesocyclops ogunnus Onabamiro, 1957 (Copepoda Cyclopoida) in water bodies of São Paulo state, identified as Mesocyclops kieferi Van de Velde, 1984. Brazilian Journal of Biology, v. 62, n. 4, p. 615-620, 2002. http://dx.doi.org/10.1590/S1519-69842002000400009

MATSUMURA-TUNDISI, T.; TUNDISI, J.G. Checklist dos Copepoda Calanoida de água doce do Estado de São Paulo. Biota Neotropica, v.11, supl.1, 2011. http://dx.doi.org/10.1590/S1676-06032011000500022

MELÃO, M.G.G. Desenvolvimento e aspectos reprodutivos de cládoceros e copépodos de águas continentais brasileiras. In: POMPÊO, M. L. M. (ed.). Perspectivas na Limnologia do Brasil. São Luís: Gráfica e Editora União, 2010. 198p. 
MELO, A. S.; HEPP, L. U. Ferramentas estatísticas para análises de dados provenientes de biomonitoramento. Oecologia Brasiliensis, v.12, n.3, p. 463-486, 2008. http://dx.doi.org/10.4257/oeco.2008.1203.07

MEIRINHO, P.A.; NISHIMURA, P.Y.; PIRES-ZOTTARELLI, C.L.A.; MOSCHINICARLOS, V.; POMPÊ, M.L.M. Olpidium gregarium, a chytrid fungus affecting rotifers populations in Rio Grande Reservoir, São Paulo State, Brazil. Biota Neotropical, v. 13, n. 3, 2013. http://dx.doi.org/10.1086/283056

MILligAN, G.W.; COOPER, M.C. A Study of Standardization of Variables in Cluster Analysis. Journal of Classification, v. 5, p.181-204, 1988. http://dx.doi.org/10.1007/BF01897163

MORTARI, R.C. Distribuição Espaço-Temporal de Cladocera (Crustacea, Branchiopoda) em uma lagoa subtropical lateral ao Rio Paranapanema (zona de desembocadura na Represa de Jurumirim/SP). 2004. 172p. Tese (Doutorado) - Instituto de Biociências, Universidade Estadual Paulista Júlio de Mesquita Filho, Botucatu, 2004.

NETTO, N.P.; BARROS, L.A.A.B. Uma história visual da construção do Sistema Cantareira. São Paulo: Ed. Autor, 2010.

NOGRADY, T.; SEGERS, H. Rotifera: Asplanchnidae, Gastropodidae, Lindiidae, Microcodidae, Synchaetidae, Trochosphaeridae and Filinia. In: DUMONT, H. J. (ed). Guides to the Identification of the microinvertebrates of the continental waters of the world. Netherlands: SPB Academic Publishers, 2002. v. 6, p.264.

NOGRADY, T.; POURRIOT, R.; SEGERS H. Rotifera: The Notommatidae and the Scaridiidae. In: DUMONT, H. J. (ed). Guides to the Identification of themicroinvertebrates of the continental waters of the world. Netherlands: SPB Academic Publishing, 1995. v. 3, 248p.

NORDI, N.; WATANABE, T. Nota preliminar sobre rotíferos (zooplancton) do Açude Epitácio Pessoa, Boqueirão, Paraíba. Revista Nordestina de Biologia, v.1, n.1, p. 31-39, 1978.

OLIVEIRA, P.E.; GOMES, A.R.; SANCHES, R.C.; SAAD, A.R. Análise da evolução da paisagem no entorno da represa dos rios Jaguari e Jacareí, estado de São Paulo, com base em sensoriamento remoto e SIG. Geociências, v.27, n.4, p.527-539, 2008.

ORTEGA-MAYAGOITIA, E.; CIROS-PEREZ, J.; SANCHEZ-MARTINEZ, M. A story of famine in the pelagic realm: temporal and spatial patterns of food limitation in rotifers from an oligotrophic tropical lake. Journal Plankton Research, v. 33, n. 1, p. 15741585, 2011. https://doi.org/10.1093/plankt/fbr045

PANOSSO, R.; CARLSSON, P.; KOZLOWSKY-SUZUKI, B.; AZEVEDO, S.M.F.O.; GRANÉLI, E. Effect of grazing by a neotropical copepod, Notodiaptomus, on a natural cyanobacterial assemblage and on toxic and non-toxic cyanobacterial strains. Journal of $\begin{array}{lllll}\text { Plankton Research, } & \text { v.25, n.9, p. } & 1169-1175,\end{array}$ https://doi.org/10.1093/plankt/25.9.1169 
PARANHOS, J. D. N.; ALMEIDA, V. L. S.; SILVA FILHO, J. P.; PARANAGUÁ, M. N.; MELO JÚNIOR, M.; NEUMANN-LEITÃO, S. The zooplankton biodiversity of some freshwater environments in Parnaíba basin (Piauí, Northeastern Brazil). Brazilian Journal of Biology, v. 73, n.1, p. 125-134, 2013. http://dx.doi.org/10.1007/s10452-0079151-x

PERBICHE-NEVES, G.; BOXSHALL, G.A.; PREVIATTELli, D.; NOGUEIRA, M.G.; ROCHA C.E.F. Identification guide to some Diaptomid species (Crustacea, Copepoda,Calanoida, Diaptomidae) of "de la Plata" River Basin (South America), Zookeys, v. 497, p. 1-111, 2015. http://dx.doi.org/10.3897/zookeys.497.8091

PEREIRA, A. P. S.; VASCO, A. N. do; BRITTO, F. B.; MÉLLO JÚNIOR, A. V.; NOGUEIRA, E. M. de S. Biodiversidade e estrutura da comunidade zooplanctônica na Sub-bacia Hidrográfica do Rio Poxim. Revista Ambiente e Água, v. 6, p. 191-205, 2011. http://dx.doi.org/10.4136/ambi-agua.194

REID, J. W.; PINTO-COELHO; R. M.; GIANI, A. Uma apreciação da fauna de copépodos (Crustacea) da região de Belo Horizonte, com comentários sobre espécies de Minas Gerais. Acta Limnologica Brasiliensia, v. 11, p. 527-547, 1988.

ROCHA, O.; MATSUMURA-TUNDISI, T.; ESPÍNDOLA, E. L. G.; ROCHE, K. F.; RIETZLER, A. C. Ecological theory applied to reservoir zooplankton, pp.457-476. In: TUNDISI, J.G.; STRAŠKRABA, M. (eds.). Theoretical reservoir ecology and its applications. São Carlos: International Institute of Ecology; Backhuys Publishers, 1999.

ROCHA, O.; SANTOS-WISNIEWSKI, M. J.; MATSUMURA-TUNDISI, T. Checklist de Cladocera de água doce do Estado de São Paulo. Biota Neotropica, v.11, p. 1-21, 2011. http://dx.doi.org/10.1590/S1676-06032011000500024

SANTOS, T.G. dos; GUSMÃO, L.M.O; NEUMANN-LEITÃO, S.; CUNHA, A.G. da. Zooplâncton como indicador biológico da qualidade ambiental nos estuários dos rios Carrapicho e Botafogo, Itamaracá-PE. Revista Brasileira Engenharia de Pesca, v. 4, n.1, p. 44-56, 2009.

SANTOS-WISNIEWSKI, M. J.; ROCHA, O. Spatial distribution and secondary production of Copepoda in a tropical reservoir: Barra Bonita, SP, Brazil. Brazilian Journal of Biology, v.67, n.2, p.223-233, 2007. http://dx.doi.org/10.1590/S1519-69842007000200007

SARTORI, L.P.; NOGUEIRA, M.G.; HENRY, R.; MORETTO, E.M. Zooplankton fluctuations in Jurumirim Reservoir (São Paulo, Brazil): a three-year study. Brazilian Journal of Biology, v. 69, n. 1, p. 1-18, 2009. http://dx.doi.org/10.1590/S151969842009000100002

SEGERS, H.; DUMONT, H. J. 102+ rotifer species (Rotifera: Monogononta) in Broa reservoir (SP., Brazil) on 26 August 1994, with the description of three new species. Hydrobiologia, v. 316, p.183-197, 1995. http://dx.doi.org/10.1007/BF00017436

SEGERS, H. Rotifera: the Lecanidae (Monogonta) In: DUMONT, H. J. (ed). Guides to the identification of the microinvertebrates of the continental waters of the world. Netherlands: SPB Academic Publishing, 1995. v. 2. 
SENDACZ, S.; CALEFFI, S.; SANTOS-SOARES, J. Biomassa de organismos zooplanctônicos em represas de diferentes condições tróficas do Estado de São Paulo, Brasil. Brazilian Journal of Biology, v.66, n.1, p.337-350, 2006. http://dx.doi.org/10.1590/S1519-69842006000200016

SENDACZ, S.; KUBO, E. Copepoda (Calanoida e Cyclopoida) de reservatórios do Estado de São Paulo. Boletim do Instituto de Pesca, n. 9, p.51-89, 1982.

SHIEL, R. J.; KOSTE, W. Rotifera from australian inland waters VIII. Trichocercidae (Monogononta). Transactions of the Royal Society of Australian, v. 116, n. 1, p.127,1992 .

SHIMABUKURO, E. M. A fauna bentônica na represa Jurumirim e lagoas marginais. In: HENRY, R. Represa de Jurumirim: Ecologia, Modelagem e Aspectos sociais. Ribeirão Preto: Holos, 2014. 435p.

SILVA, W.M. Diversidade dos Cyclopoida (Copepoda, Crustacea) de água doce do Estado de São Paulo: taxonomia, ecologia e genética. 2003. 170f. Tese (Doutorado em Ecologia e recursos naturais) - Universidade Federal de São Carlos, São Carlos, 2003.

SILVA, W. M.; MATSUMURA-TUNDISI, T. Checklist dos Copepoda Cyclopoida de vida livre de água doce do Estado de São Paulo, Brasil. Biota Neotropica, v.11, supl.1, 2011. http://dx.doi.org/10.1163/156854001750243054

SOUZA-SOARES, F.; TUNDISI, J. G.; MATSUMURA-TUNDISI, T. M. Checklist of freshwater Rotifera from São Paulo State, Brazil. Biota Neotropica, v. 11, n.1a, 2011. http://dx.doi.org/10.1590/S1676-06032011000500020

SORF, M.; DEVETTER, M. Coupling of seasonal variations in the zooplankton community within the limnetic and littoral zones of a shallow pond. International Journal of Limnology, v.47, n.3, p-259-268, 2011. https://doi.org/10.1051/limn/2011006

TEIXEIRA, C.; TUNDISI, J. G.; KUTNER, M. B. Plankton studies in a mangrove II. The standing stock and some ecological factors. Boletim Instituto Oceanográfico, v. 24, p.23-41, 1965. http://dx.doi.org/10.1590/S0373-55241965000100002

THORNTON, K. E.; KIMMEL, B. L.; PAYNE, F. E. Reservoir Limnology: Ecological perpectives. New York: John Wiley and Sons, 1990. 246p.

TUNDISI, J. G.; MATSUMURA-TUNDISI, T. Limnologia. São Paulo: Oficina de Textos, 2008, 632p.

VALDERRAMA, J. C. The simultaneous analysis of total nitrogen and total phosphorus in natural waters. Marine chemistry, v.10, p.109-222, 1981. https://doi.org/10.1016/03044203(81)90027-X

XIE, P.; IWAKUMA, T.; FUJII, K. Changes in the structure of a zooplankton community during a Ceratium (dinoflagellate) bloom in a eutrophic fishless pond. Journal of $\begin{array}{llllll}\text { Plankton Research, } & \text { v. } & \text { 20, n.9, }\end{array}$ https://doi.org/10.1093/plankt/20.9.1663

WHATELY, M.; CUNHA, P. Cantareira 2006: um olhar sobre o maior manancial de água da Região Metropolitana de São Paulo. São Paulo: Instituto Sócio-Ambiental, 2007. 68p. 